\title{
An unusual structure near the porta hepatis: What's your diagnosis?
}

A 87 -year-old man with Achronic obstructive pulmonary disease and asbestosis experienced progressive weight loss, asthenia, dyspnea and increased productive cough in May 2003. He appeared chronically ill. CT imaging of the lung and diagnostic bronchoscopy failed to disclose the cause, but in June 2003 sputum culture grew $M y$ cobacteria. Standard treatment for tuberculosis was commenced.

When Mycobacterium aviumintracellulare was isolated, atypical mycobacterial infection of the upper lobe was diagnosed. The patient's drug therapy was converted to ethambutol, rifampin and clarithromycin (the latter was eventually replaced by ciprofloxacin and clofazimine).

Repeat chest CT and abdominal CT scans were performed in August, since the patient's condition had not improved. The scans revealed lung parenchymal changes consistent with $M$. avium-intracellulare infection and an infrarenal aortic aneurysm, but no malignant disease. A lowdensity mass $6 \times 4 \mathrm{~cm}$ in diameter that contained areas of coarse calcification was observed at the inferior aspect of the head of the pancreas. The same mass had been found on a CT scan performed 4 years previously, and it had been reported as possibly representing a pancreatic pseudocyst.

The patient's condition continued to decline, and he was admitted to hospital. In December 2003 a CT scan again revealed a low-density, fluid-attenuating structure $6 \times 4 \mathrm{~cm}$ in diameter that was anterolateral to the inferior vena cava and near the porta hepatis (Fig. 1). It was interpreted $\propto$ as either a pancreatic pseudocyst $\vec{q}$ or a lymph node compatible with disseminated M. avium-intracellulare. Despite ongoing quadruple antibiotic therapy, the patient died 12 days later.

At autopsy 2 days after death, we found severe cavitation and
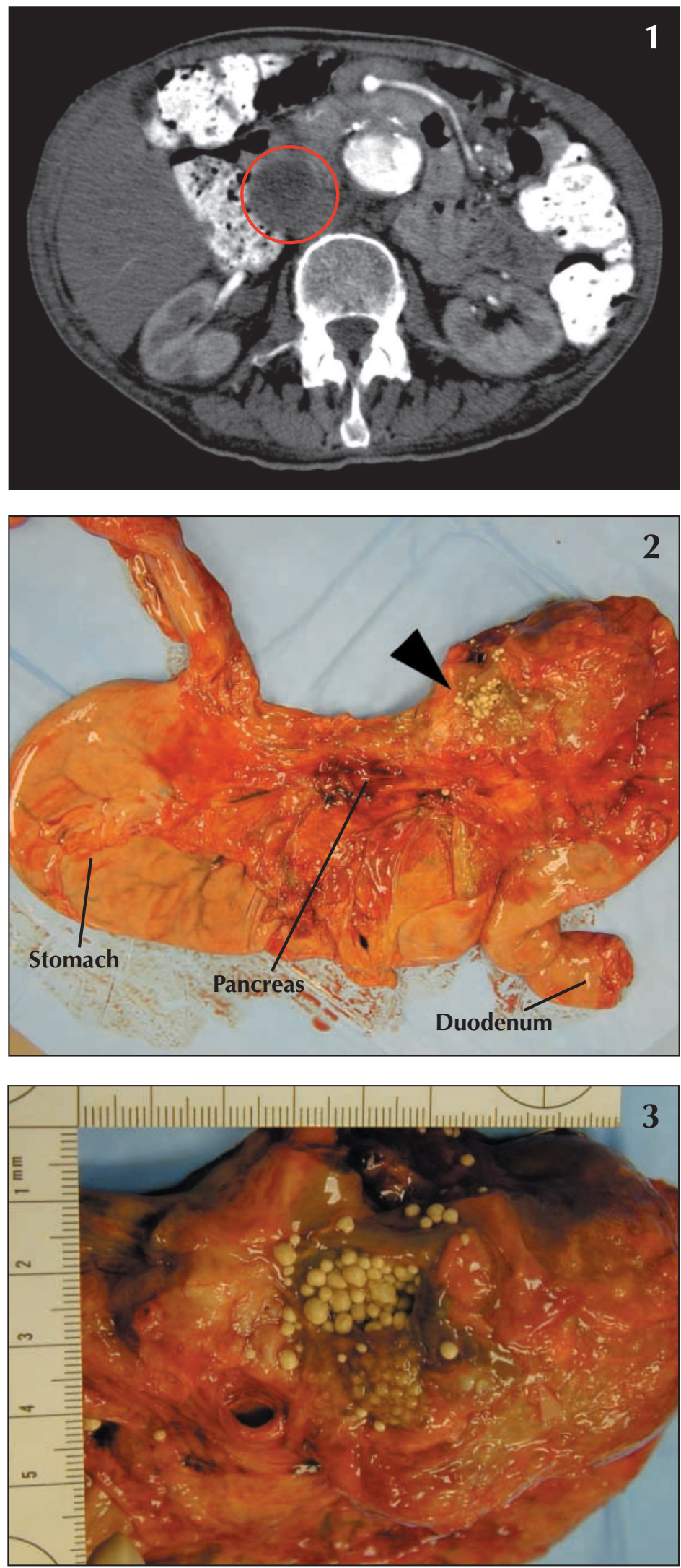
fibrosis of the lungs consistent with chronic $M$. avium-intracellulare infection, atherosclerotic cardiovascular disease and an unruptured $6-\mathrm{cm}$ infrarenal aortic aneurysm.

An unexpected finding was a smooth and shiny multiloculated cystic lesion adjacent to the head of the pancreas (Fig. 2, arrow). This measured $7 \mathrm{~cm}$ at its widest point and was filled with a greyish-brown fluid that contained numerous spherical objects $2-3 \mathrm{~mm}$ in diameter. The spheres were soft, noncalcified and grey-white with a texture like soft clay. Some of the cysts were filled with a white-gray pasty material, and in others clear liquid bathed the spheres (Fig. 3). The remainder of the pancreas was unremarkable.

This lesion was unidentifiable at the time of autopsy. Several experienced pathologists invited to review the fresh material were unable to offer a diagnosis.

Standard bacteriologic, fungal and mycobacterial cultures of the unusual material grew no organisms. Microscopy of the cystic mass showed a lining composed of flattened epithelial cells surrounded by lymphocytes with occasional germinal centres and foamy histiocytes. In some areas the epithelium was absent, and foreign-body granulomas were present. The cyst contents were composed of acellular keratotic debris and cholesterol crystals (Figs. 4 and 5).

\section{Victor Meneghetti}

Medical Student

Charles Lee

Department of Pathology

Thomas L. Perry

Department of Medicine

Gordon Andrews

Department of Radiology

University of British Columbia

Vancouver, BC
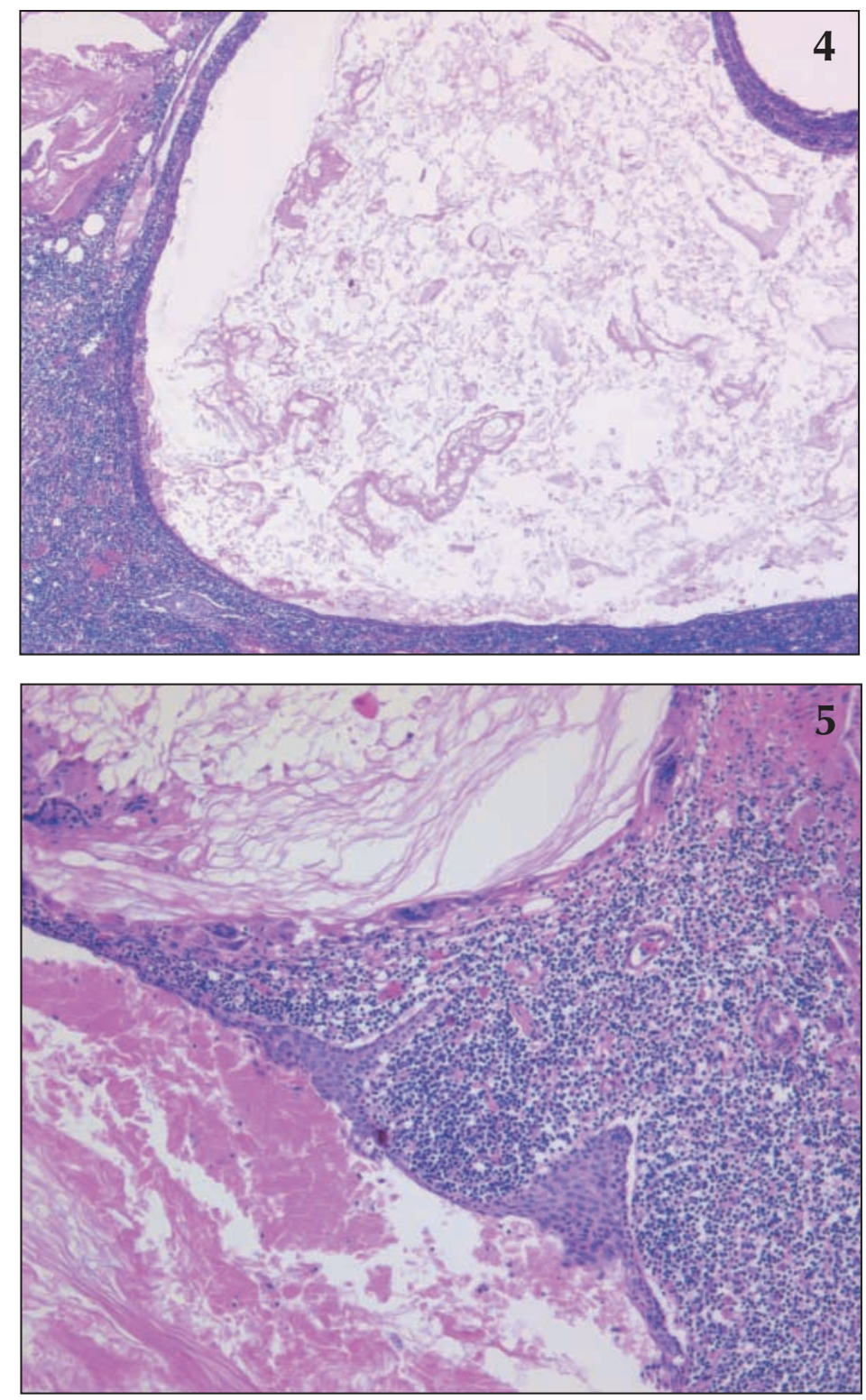

\section{What's your diagnosis?}

We invite you to submit your ideas about the cause of these lesions by sending an eLetter response to this article at www .cmaj.ca. We will compile the best letters and print them in summary in an upcoming issue.

Do you have a case that you can't diagnose? Send a description of it to the attention of Dr. Wooltorton at eric.wooltorton@ cma.ca. 